\title{
Diminution de l'expression du gène de la glycérophosphate déshydrogénase par la dexaméthasone, dans les cellules 3T3-F442A différenciées : antagonisme avec l'insuline et l'antiglucocorticoïde RU38486
}

\author{
N Moustaïd 1, B Hainque 1, A Quignard-Boulange $1^{*}$, J Pairault 2 \\ 1 INSERM U177 15, rue de l'École de médecine 75006 Paris; \\ 2 INSERM U282, hôpital Henri Mondor 92400 Créteil, France
}

(15 réunion du groupe Développement, INRA, Paris, 24-26 mai 1989)

\begin{abstract}
Résumé - La différenciation des préadipocytes de la lignée 3T3 se caractérise par l'augmentation de l'activité de plusieurs enzymes du métabolisme lipidique. Le rôle de la glycérophosphate déshydrogénase (G3PDH), enzyme clé de la lipogenèse, a été particulièrement étudié. Dans ce travail, nous avons étudié la régulation hormonale de la G3PDH dans les cellules 3T3-F442A différenciées.

La dexaméthasone (DEX) diminue de $50 \%$ le taux d'ARNm ainsi que l'activité spécifique de la G3PDH. La DEX régule l'expression de cette enzyme à un niveau post-transcriptionnel en augmentant la dégradation du messager; la transcription du gène n'étant pas modifí́e. La DEX contrôle spécifiquement l'expression du gène de la G3PDH, puisque celle d'un autre marqueur de la différenciation adipocytaire, l'adipsine, n'est pas modifiée par la DEX. L'insuline s'oppose à l'effet inhibiteur de la DEX en stabilisant le messager de la G3PDH. L'antiglucocorticoïde RU38486 supprime l'effet de la DEX; ceci suggérerait que l'action de la DEX est médiée par les récepteurs des glucocorticoïdes.
\end{abstract}

préadipocyte 3T3 / glycérophosphate deshydrogénase / Insuline / dexaméthasone / antiglucocorticoïde

Summary - Decreased gene expression of glycerophosphate dehydrogenase by dexamethasone in 3T3-F442A adipocytes: insulin and antiglucocorticoid RU38486 antagonism. Preadipocyte subclones derived from mouse 3T3 cells differentiate into adipocytes; this differentiation is characterized by an increased activity of numerous enzymes required for triglyceride synthesis and/or mobilization. Among these enzymes, the role of glycerophosphate dehydrogenase in the differentiation process has been previously reported.

In the present work, we studied the hormonal regulation of glycerophosphate dehydrogenase gene expression (G3PDH) in differentiated 3T3-F442A adipocytes. Dexamethasone (DEX) elicited a $50 \%$ decrease in both mRNA content and specific activity of G3PDH. This effect was due to a posttranscriptional event since DEX shortened the half life of the mRNA, whereas it did not modify the transcription rate of this gene. The DEX effect is specific to G3PDH, since the expression of another adipose-specific gene, namely adipsin, is not modified by DEX treatment. Insulin counteracts the in-

\footnotetext{
* Correspondance et tirés à part
} 
hibitory effect of DEX, mainly by stabilizing the MRNA encoding for G3PDH. The antiglucocorticoid RU38486 is able to reverse DEX inhibition. Latter phenomenon suggests that DEX action on G3PDH gene expression could be mediated by glucocorticoid receptors.

3T3 preadipocyte / glycerophosphate dehydrogenase / insulin / dexamethasone / antiglucocorticold

\section{INTRODUCTION}

La différenciation in vitro des cellules de la lignée préadipocytaire 3T3-F442A aboutit à la formation de cellules dont les caractéristiques morphologiques et les fonctions métaboliques sont comparables à celles d'adipocytes matures (Green et Kehinde, 1976; Green, 1978). Le processus de différenciation est caractérisé par l'apparition séquentielle d'activités enzymatiques de la lipogenèse et par le développement de la sensibilité et de la réponse aux hormones lipogéniques et lipolytiques (Green, 1978; Rubin et al, 1978). Parmi ces enzymes qui sont considérées comme des marqueurs de la différenciation, la glycérophosphate déshydrogénase (G3PDH), ainsi que l'adipsine, ont des concentrations très augmentées au cours de la conversion adipocytaire (Wise et Green, 1979; Spiegelman et al, 1983). Nous avons précédemment montré que la dexaméthasone (DEX) inhibe la différenciation terminale des cellules 3T3-F442A et, en particulier, l'activité de la G3PDH, qui est une enzyme clé de la lipogenèse (Pairault et Lasnier, 1987; Moustaïd et al, 1988).

Dans ce travail, nous avons recherché une éventuelle régulation de l'expression du gène de la G3PDH dans les cellules 3T3-F442A en phase de maturation terminale. Nos résultats montrent que ce gène est régulé de manière négative et à un niveau post-transcriptionnel par la dexaméthasone; cet effet de la DEX est annihilé par l'insuline.
Le traitement des cellules par un antiglucocorticoïde, le RU486 (Agarwal et al, 1987), supprime l'effet de la DEX sur l'expression du gène de la G3PDH, ce qui suggère que l'action de la DEX est médiée par les récepteurs des glucocorticoïdes.

\section{MATÉRIELS ET MÉTHODES}

\section{Cultures cellulaires}

Les cellules 3T3-F442A, fournies par le $\mathrm{Dr} H$ Green (Harward Medical School, Boston) sont maintenues comme précédemment décrit (Pairault et al, 1982). Les cellules sont cultivées jusqu'à la confluence dans du DMEM (Dulbecco's Modified Eagle Medium) supplémenté en sérum de veau fœetal (10\%), puis différenciées pendant $12 \mathrm{j}$ dans le même milieu contenant de l'insuline (10 $\left.\mathrm{nmol} . \mathrm{I}^{-1}\right)$. Les cellules sont alors traitées ou non par DEX (10 nmol.t-1) et-ou l'insuline bovine (10 nmol..$\left.^{-1}\right)$. L'effet de l'antiglucocorticoïde RU486 (fourni par Roussel-Uclaf) a été également testé en traitant les cellules différenciées avec de la DEX en présence ou non de la drogue. Les dosages des activités enzymatiques et l'analyse par northern-blot des ARN totaux sont effectués aux $j$ indiqués sur les figures.

\section{Dosage enzymatique}

Les tapis cellulaires sont suspendus dans le tampon sucrose $250 \mathrm{mmol.1} \mathrm{I}^{-1}$, contenant du DTT (1 mmol. $\mathrm{I}^{-1}$ ) et de I'EDTA (1 mmol. $\left.\mathrm{I}^{-1}\right)$, comme précédemment décrit (Moustaïd et al, 1988). L'activité G3PDH est déterminée sur le surnageant $100000 \mathrm{~g}$ selon la technique de Wise et Green (1979). Les protéines sont do- 
sées par la méthode de Bradford (1976) en utilisant la sérum albumine bovine comme standard.

\section{Extraction et analyse par northern-blot des ARN totaux}

Les ARN totaux sont extraits selon la technique de Cathala et al (1983). L'analyse par northernblot de ces ARN est effectuée comme précédemment décrit (Dugail et al, 1988) à l'aide des sondes ADNc (pGPD1, p $\beta$-actine et pAD20). Ces sondes ADNc reconnaissent 1 espèce moléculaire d'ARNm de 3,5, 2,15 et 1,05 kb respectivement pour la G3PDH, l'actine et l'adipsine (Spiegelman et al, 1983). Afin de tenir compte des différences entre les quantités d'ARN déposées et l'efficacité du transfert, les résultats des northern-blot sont quantifiés par densitométrie et normalisés par rapport au signal du messager $\beta$-actine.

\section{Stabilité de I'ARNm de la G3PDH}

Les adipocytes 3T3-F442A sont traités ou non pendant $14 \mathrm{~h}$ par la dexaméthasone, en présence ou en absence d'insuline. La synthèse des ARNm est ensuite inhibée par addition de $25 \mu \mathrm{g} / \mathrm{ml}$ de 5,6 dichloro 1- $\beta-\mathrm{D}$ ribofuranosyl benzimidazole (Sehgal et al, 1976). Les ARN sont extraits à différents temps de traitement par la drogue puis analysés par northern-blot. La demi-vie du messager est estimée après quantification des résultats.

\section{RÉSULTATS}

Dans les cellules pleinement différenciées, la présence d'insuline n'est pas un facteur nécessaire au maintien à un niveau élevé de l'activité G3PDH puisque celle-ci n'est pas modifiée après $3 \mathrm{j}$ de privation d'insuline (fig 1). Par contre, l'activité G3PDH diminue de $50 \%$ après $48 \mathrm{~h}$ de traitement

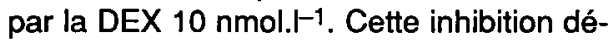

pendante de la dose est maximale à la concentration de $10 \mathrm{nmol}^{-1}{ }^{-1}$ (fig 1 et 2). L'effet de la DEX peut être reproduit par l'hormone naturelle, la corticostérone, mais à des concentrations plus élevées (fig 2). En présence d'insuline, la DEX ne modifie pas significativement l'activité pendant les 2 premiers j; après $3 \mathrm{j}$ de traitement, l'activité est diminuée de $20 \%$.

Nous avons voulu savoir si cet effet était lié à une diminution du taux d'ARNm codant pour cette enzyme. La figure 3 montre que le messager G3PDH, indétectable dans les préadipocytes (P), est fortement exprimé dans les adipocytes (A). La présence d'insuline n'exerce qu'un faible effet positif sur lo messager de la G3PDH $(+25 \%)$ tandis qu'elle provoque une diminution de $70 \%$ du messager d'un autre marqueur de différenciation, l'adipsine. Par contre, la DEX diminue de moitié le taux d'ARNm de la G3PDH après $24 \mathrm{~h}$ de traitement : cet effet de la DEX est spécifique de la G3PDH puisque le messager de l'adipsine, dans les mêmes conditions, n'est pas modifié (fig 3). Comme pour l'activité enzymatique, la présence d'insuline contrecarre l'effet inhibiteur de la DEX (fig 2 et 3).

Nous avons également étudié la spécificité d'action de la DEX et son éventuelle reversibilité par l'antiglucocorticoïde RU486; nous montrons que le traitement associant la DEX et le RU486 ne modifie ni l'activité enzymatique (fig 4), ni le taux du messager de la G3PDH (fig 5).

La quantité d'ARNm codant pour la G3PDH peut être affectée par sa vitesse de dégradation, et nous avons, dans ce but, étudié l'effet de la DEX sur la stabilité du messager de la G3DPH. Après quantification par densitométrie, les résultats montrent que la présence de la DEX accélère la dégradation de cet ARNm puisque sa demi-vie passe de 7,2 h à 4,5 h. Par 
A

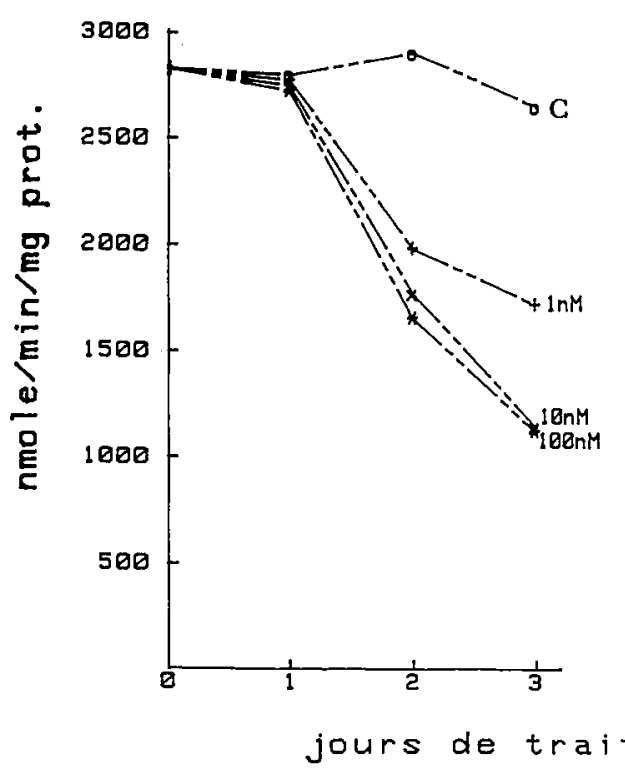

B

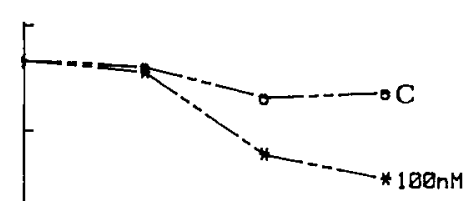

Flg 1. Effet de la DEX sur l'activité G3PDH. Les cellules 3T3-F442A différenciées sont maintenues et traitées pendant $3 \mathrm{j}$ par la DEX, en absence (A) ou en présence (B) d'insuline $10 \mathrm{nmol} . \mathrm{I}^{-1}$. La DEX est ajoutée ou non (C : contrôle) dans les milieux de culture aux doses : 1,10 ou 100 nmol..l-1. L'activité G3PDH est dosée dans le surnageant $100000 \mathrm{~g}$ des extraits cellulaires et exprimée en nmoles de NADH oxydées par min et par mg de protéines.

contre, le maintien d'insuline entraîne une stabilisation du messager ( $>20 \mathrm{~h}$ ) et s'oppose à l'effet de la DEX (15 h).

\section{DISCUSSION}

Des études récentes ont montré que les glucocorticoïdes accélèrent l'expression de certains gènes spécifiques au cours de la différenciation des préadipocytes TA1/2 (Chapman et al, 1984; Ringold et al, 1988). Dans les cellules 3T3-L1, la DEX associée à l'insuline et à la méthylisobutylxanthine est utilisée comme inducteur de la différenciation (Rubin et al, 1978). Dans les cellules 3T3-F442A, nous avons précédemment montré que la DEX inhibe l'expression d'enzymes lipogéniques, en particulier la G3PDH, au cours de la conversion adipocytaire (Pairault et Lasnier, 1987; Moustaïd et al, 1988). Dans ce travail, nous avons étudié la régulation de l'expression de ce gène par les glucocorticoïdes dans les cellules 3T3-F442A pleinement différenciées. Nos résultats montrent que la diminution de l'activité enzymatique par la DEX peut être reliée à une diminution du taux d'ARNm codant pour cette enzyme. La diminution de l'expression du gène codant pour la G3PDH peut être attribuée à un effet transcriptionnel ou posttranscriptionnel. Des expériences de transcription in vitro réalisées sur des noyaux 


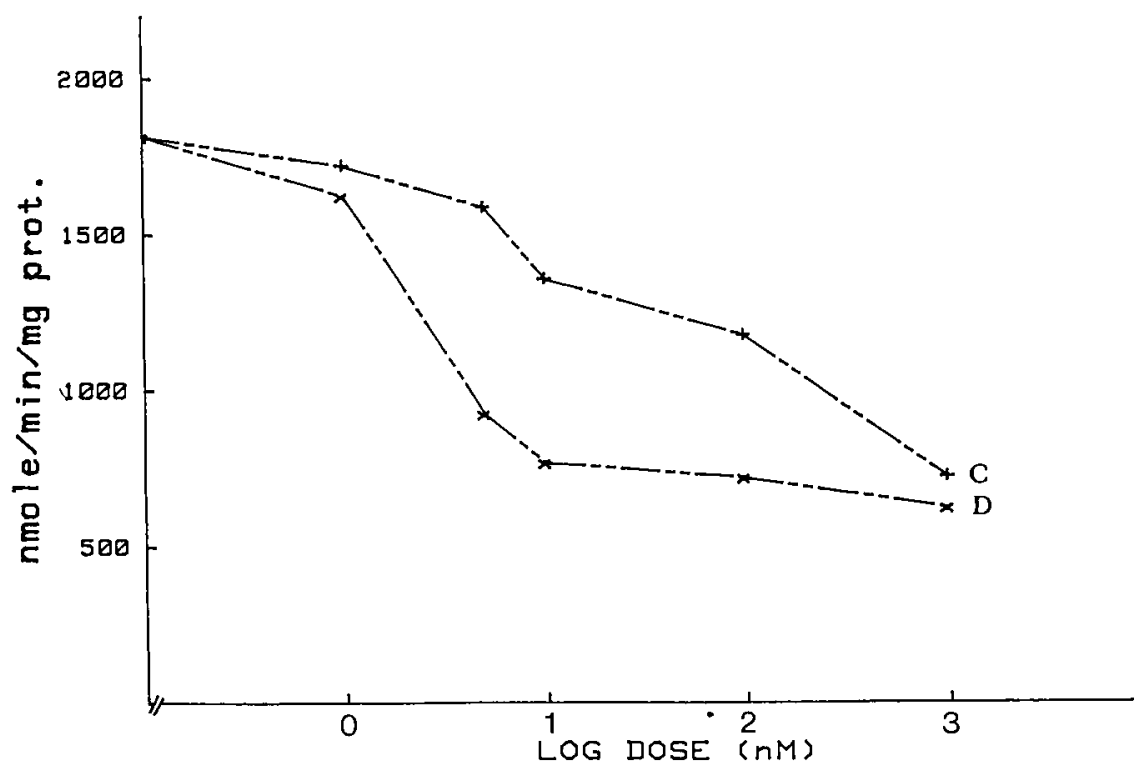

Fig 2. Effets de la DEX et de la corticostérone sur l'activité G3PDH. Les cellules différenciées sont traitées par la DEX (D) ou la corticostérone (C) aux doses indiquées. L'activité G3PDH est ensuite mesurée comme décrit ci-dessus, après 2 j de traitement hormonal.

provenant de cellules traitées ou non par la DEX montrent que la capacité de transcription du gène de la G3PDH est identique dans les cellules traitées ou non par la DEX (Moustaïd et al, 1990). Ainsi, la DEX semble agir à un niveau posttranscriptionnel en augmentant la dégradation du messager G3PDH. Cet effet peut être lié à une activation de ribonucléases spécifiques des messagers, ou à une augmentation de la synthèse de ces ribonucléases par ces hormones (Gessani et al, 1988; Simonet et Ness, 1989). II semble pour la G3PDH, que l'hypothèse d'une augmentation de synthèse des ribonucléases soit peu probable car le traitement des cellules par la cycloheximide ne modifie pas l'effet de la DEX (résultats non présentés). Cet effet inhibiteur de la DEX sur des adipocytes en culture est étayé par des études effectuées sur le tissu adipeux montrant que les glucocorticoïdes diminuent l'activité de certaines enzymes lipogéniques telles que la synthétase des acides gras et l'acétyl CoA carboxylase (Volpe et Marasa, 1975). Dans certains cas, l'activité de la lipoprotéine lipase est également diminuée par la DEX dans ce tissu (Bagdade et al, 1976). Plus récemment, il a été montré que l'expression du gène d'une autre enzyme, la phosphoénolpyruvate carboxykinase est inhibée par la DEX et ce, essentiellement à un niveau post-transcriptionnel (Nechushtan et al, 1987). Par ailleurs, l'effet inhibiteur de la DEX est spécifique de la G3DPH puisqu'il n'y a pas de modification de l'expression de l'adipsine, un autre marqueur de la différenciation. Cette situation est à l'opposé de celle observée dans le cas de cellules 


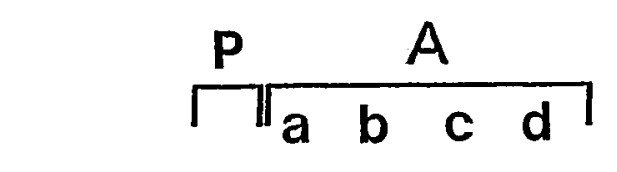

$3.5 \mathrm{~kb}$

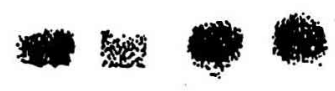

$1 \mathrm{~kb}$

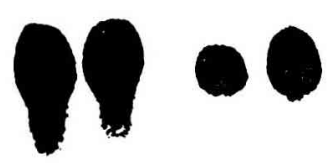

Fig 3. Effet de la DEX sur les messagers de la G3PDH et de l'adipsine. Les ARN totaux sont extraits des préadipocytes $(P)$ ou des adipocytes (A). $40 \mu \mathrm{g}$ de ces ARN sont ensuite analysés par northern-blot comme indiqué dans les Matériels et Méthodes. Les pistes sont définies comme suit : a : contrôle; b : DEX 10 nmol..$^{-1}$; $c$ : insuline $10 \mathrm{nmol} . \mathrm{I}^{-1} ; \mathrm{d}$ : insuline $10 \mathrm{nmol} . \mathrm{I}^{-1}+$ DEX $10 \mathrm{nmol}^{-1} \mathrm{I}^{-1}$. Les signaux obtenus correspondent respectivement aux messagers de la G3PDH (3,5 kb) et de l'adipsine (1 kb).

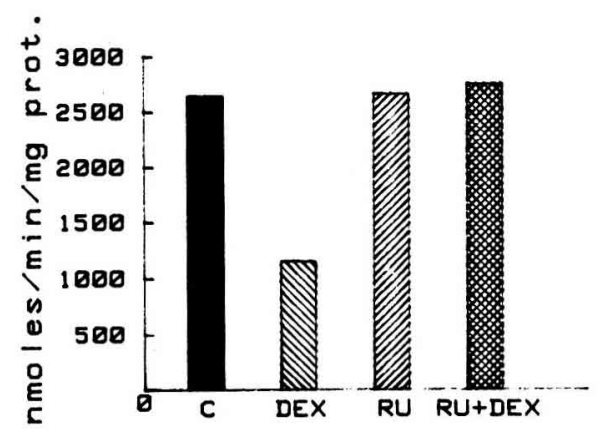

Fig 4. Effet du RU486 sur l'activité G3PDH. Les adipocytes 3T3-F442A sont traités ou non (C) pendant 3 j avec $10 \mathrm{nmol} / /^{-1}$ de dexaméthasone (DEX) et-ou $10 \mathrm{nmol} / /^{-1}$ de RU38486 (RU). L'activité enzymatique est ensuite mesurée sur le surnageant $100000 \mathrm{~g}$ pour les différents traitements.

\section{D R RD}

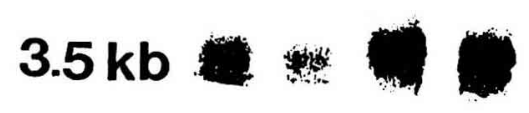

Fig 5. Effets du RU486 sur l'ARNm de la G3PDH. $40 \mu \mathrm{g}$ des ARN totaux extraits des cellules, traitées comme indiqué ci-dessus, sont analysés par northern-blot. Les pistes correspondent aux traitements suivants : $\mathrm{C}$ : contrôle; D : DEX $10 \mathrm{nmol} / /^{-1} ; \mathrm{R}$ : RU486 $10 \mathrm{nmol} / \mathrm{l}^{-1}$; $\mathrm{RD}$ : DEX $10 \mathrm{nmol} / /^{-1}+\mathrm{RU} 48610 \mathrm{nmol} / /^{-1}$.

traitées par l'insuline; l'expression de l'adipsine est fortement réduite en présence d'insuline, ce qui est en accord avec les résultats obtenus par Dani et al (1989) dans la lignée Ob17.

Récemment, Dobson et al (1987) n'ont pas trouvé d'effet de la DEX sur l'ARNm de la G3PDH. D'après nos résultats qui montrent un antagonisme entre la DEX et l'insuline, cette absence d'effet de la DEX pourrait s'expliquer par la présence de fortes doses d'insuline dans les milieux de culture utilisés par ces auteurs.

Nous avons précédemment mis en évidence des récepteurs spécifiques des glucocorticoïdes dans les cellules 3T3-F442A (Hainque et al, 1987). Dans cette étude, nous montrons que l'antiglucocorticoïde RU486 supprime l'effet inhibiteur de la DEX sur l'expression du gène de la G3PDH. Ainsi, nos résultats suggèrent que la liaison de l'hormone aux récepteurs des glucocorticoïdes est une étape dans l'action de la DEX.

En conclusion, nous montrons dans ce travail que la DEX inhibe l'expression du gène de la G3PDH, une enzyme clé dans la lipogenèse, en augmentant la dégradation de son ARNm; l'insuline s'oppose à cet effet. 
L'ensemble des résultats suggère que les glucocorticoïdes contrôlent activement le métabolisme lipidique du tissu adipeux et que l'importance de leur contribution à la régulation de la masse adipeuse dépend de l'état insulinique du sujet.

\section{RÉFÉRENCES}

Agarwal MK, Hainque B, Moustaiid N, Lazar G (1987) Glucocorticoid antagonists. FEBS Lett 217, 221-226

Bagdade JD, Yee E, Albers J, Pykalisto OJ (1976) Glucocorticoids and triglyceride transport: effects on triglyceride secretion, rates, lipoprotein lipase and plasma lipoproteins in the rat. Metabolism 25, 533-542

Bradford M (1976) A rapid and sensitive method for the quantification of microgram quantities of protein utilizing the principle of protein-dye binding. Anal Biochem 72, 248-254

Cathala G, Savouret JF, Mendez B, West BL, Karin M, Martial JA, Baxter JD (1983) A method for isolation of intact, translationnaly active ribonucleic acid. DNA 2, 329-335

Chapman AG, Knight DM, Dieckman BS, Ringold GM (1984) Analysis of gene expression during differentiation of adipogenic cells in culture and hormonal control of the developmental programm. $J$ Biol Chem 259, 1554815555

Dani C, Bardon S, Doglio A, Amri E, Grimaldi P (1989) Regulation of gene expression by insulin in adipose cells: opposite effects on adipsin and glycerophosphate dehydrogenase genes. Mol Cell Endocrinol 63, 199-208

Dobson DE, Groves DL, Spiegelman BM (1987) Nucleotide sequence and hormonal regulation of mouse glycerophosphate dehydrogenase mRNA during adipocyte and muscle cell differentiation. $J$ Biol Chem 262, 18041809

Dugail I, Quignard-Boulangé A, Bazin R, Lepiepvre X, Lavau M (1988) Adipose-specific increase in glyceraldehyde-3-phosphate dehydrogenase activity and mRNA amounts in suckling pre-obese Zucker rats. Biochem $J$ 254, 483-487

Gessani S, Mc Candleys S, Baglioni C (1988) The glucocorticoid dexamethasone inhibits synthesis of interferon by decreasing the level of its mRNA. J Biol Chem 263, 74547457

Green H (1978) The adipose conversion of 3T3 cells. In: Miami Winter symposium. Differentiation and development (Ahmad F, Russell TR, Schultz J, Werner R, eds) Acad Press, New York, 15, 13-36

Green H, Kehinde O (1976) Spontaneous heritable changes leading to increased adipose conversion in 3T3 cells. Cell 7, 105-113

Hainque B, Moustaïd N, Quignard-Boulangé A, Ardouin B, Lavau M (1987) Glucocorticoid binding during the differentiation of 3T3F442A fibroblasts into adipocytes. A possible regulatory effect of insulin. Biochim Biophys Acta 931, 347-353

Moustaīd N, Hainque B, Quignard-Boulangé A (1988) Dexamethasone regulation of terminal differentiation in 3T3-F442A preadipocyte cell line. Cytotechnology 1, 285-293

Moustaïd N, Lasnier F, Hainque B, QuignardBoulangé A, Pairault J (1990) Analysis of gene expression during adipogenesis in 3T3F442A preadipocytes: insulin and dexamethasone control. J Cell Biochem 42, 243-254

Nechushtan H, Benvenesty N, Brandeis R, Reshef $L$ (1987) Glucocorticoid control phosphoenolpyruvate carboxykinase gene expression in a tissue specific manner. Nucl Acid Res 15, 6405-6417

Pairault J, Lasnier F (1987) Control of the adipogenic differentiaton of 3T3-F442A cells by retinoic acid, dexamethasone, and insulin: a topographic analysis. $J$ Cell Physiol 132, 279286

Pairault J, Lasnier F, Laudat MH (1982) Développement de la stimulation de l'adénylate cyclase par l'isoprotérénol et la corticotropine $\beta^{(1-24)}$ au cours de la conversion adipocytaire des cellules 3T3-F442A en culture. Eur J Biochem 127, 351-358

Ringold GM, Chapman AB, Knight MN, Torti FM (1988) Hormonal control of adipocyte differentiation and adipocyte gene expression. Recent Prog Horm Res 44, 115-140

Rubin CS, Hirsh A, Fung C, Rosen O (1978) Development of hormone receptors and hormonal responsiveness in vitro. J Biol Chem 253, 7570-7578

Sehgal PB, Darnell JE Jr, Tamm I (1976) The inhibition of DRB (5,6-dichloro-1- $\beta$-Dribofuranosylbenzimidazole) of hRNA and 
mRNA production in Hela cells. Cell 9, 473-480

Simonet WS, Ness GC (1989) Posttranscriptional regulation of 3-Hydroxy-3Methyl Glutaryl-CoA reductase mRNA in rat liver. J Biol Chem 264, 569-573

Spiegelman BM, Frank M, Green H (1983) Molecular cloning of mRNA from $3 T 3$ adipocytes. J Biol Chem 258, 10083-10089
Volpe JJ, Marasa JC (1975) Hormonal regulation of fatty acid synthetase, acetyl-CoA carboxylase and fatty acid synthesis in mammalian adipose tissue and liver. Biochim Biophys Acta 380, 454-472

Wise LS, Green H (1979) Participation of one isozyme of cytosolic glycerophosphate dehydrogenase in the adipose conversion of 3T3 cells. J Biol Chem 254, 273-275 